\title{
Guest-editorial
}

\section{Special Issue HIS 2007}

\author{
Teresa Ludermir ${ }^{\mathrm{a}}$, Andreas König ${ }^{\mathrm{b}}$ and Andre C.P.L.F. de Carvalho ${ }^{\mathrm{c}}$ \\ ${ }^{a}$ Centro de Informática, Federal University of Pernambuco, Caixa Postal 7851, CEP 50740-540, Recife, PE, Brazil \\ E-mail:tbl@cin.ufpe.br \\ ${ }^{\mathrm{b}}$ Institute of Integrated Sensor Systems, University of Kaiserslautern, Germany \\ E-mail: koenig@eit.uni-kl.de \\ ${ }^{\mathrm{c}}$ Department of Computer Science, University of São Paulo, SCE - ICMSC - USP, Caixa Postal 668, 13560-970 \\ Sao Carlos, SP, Brazil \\ E-mail:andre@icmc.usp.br
}

This issue presents four papers selected among the best contributions to the Seventh International Conference on Hybrid Intelligent Systems (HIS'07), held in Kaiserslautern, Germany, in September 17th to 19th, with researchers from 15 countries. This seventh edition of a series of very successful conferences was technically co-sponsored by IEEE Systems Man and Cybernetics Society, Deutsches Forschungsinstitut Für Künstliche Intelligenz (DFKI), Fraunhofer Institut Techno- und Wirtschaftsmathematik (ITWM), the German Chapter of IEEE Computational Intelligence Society, the Arbeitskreis Bildanalyse und Mustererkennung Kaiserslautern (BAMEK), The World Federation on Soft Computing (WFSC), and Phytec Messtechnik $\mathrm{GmbH}$.

The conference had papers covering a large range of topics, including topics like:

Novel and hybrid methods of soft computing, neural and evolutionary computing, signal and image processing

Methodology and frameworks for automated Hybrid Intelligent Systems design

Hybrid Intelligent Systems applications Evolving/adaptive Hybrid Intelligent Systems Intelligent Sensor systems

Self-monitoring sensor systems

Organic Computing systems

Wireless intelligent sensor systems
Distributed intelligent systems/sensor networks Bio-inspired Hybrid Intelligent Systems hardware

The HIS'07 conference had an international Program Committee, with well-known researchers from 23 countries. The Program Committee selected 52 papers for oral presentation and 13 for poster presentation, based on reviews from at least two independent referees. The accepted papers were included in a proceedings by IEEE Computer Society, USA. The conference had invited talks from prestigious researchers: Prof. Takeshi Yamakawa, Dr. Yaochu Jin, Prof. Nikola Kasabov, Dr. Carlos Fonseca, and Prof. Bernt Schiele.

The authors whose works had the best reviews were invited to submit an extended version for their paper to this Special Issue. In a second selection process, new reviewers looked at the originality, significance and technical contribution of the papers. In the end, four papers were selected for this special issue. We believe that this issue presents a set of valuable papers reporting high quality work in the area of Hybrid Intelligent Systems.

The selected papers cover different aspects of Hybrid Intelligent Systems. The selected works are related with meta-learning, file sharing networks, sensor systems and extraction of probabilistic rules. Next, we briefly describe what you are going to read in these selected papers. 
The first paper, Selective Generation of Training Examples in Active Meta-Learning, by Ricardo B. C. Prudêncio and Teresa B. Ludermir, deals with reduction in the size of the set of meta-examples. The process involved with the generation of a good set of metaexamples can have a high cost. The authors combine meta-learning with active learning for the set reduction in a method they call Active Meta-Learning. Two different active learning techniques are evaluated and experiments are performed for two meta-learning tasks.

In the second paper, Hybrid Intelligent and Adaptive Sensor Systems with Improved Signal Integrity based on Dynamically Reconfigurable Matched Universal Sensor Electronics, by Senthil Kumar Lakshmanan and Andreas König, the authors investigate hybrid intelligent sensor systems using dedicated hardware for intelligent processing and system adaptation. In the paper, the authors discuss the challenges associated with the use of small components in the sensor system. In order to have an efficient and effective solution, they adapt strategies employed by sensor electronics for their use in hybrid intelligent and adaptative sensor systems.

The third paper, BayesRule: a Markov-Blanket based procedure for extracting a set of probabilistic rules from Bayesian classifiers, by Estevam R. Hruschka Jr., M. do Carmo Nicoletti, Vilma A. de Oliveira and
Gláucia M. Bressan, deals with the extraction of knowledge from Bayesian classifiers. For such, the authors propose a new approach that can make the extracted knowledge more understandable through its translation to a representation based on classification rules. The proposed method, named BayesRule, is able to reduces the number of rules and the number of antecedent conditions in the rules by using the concept of MarkovBlanket.

The last selected work, Query-Trail-Mediated Topology Reconstruction for Exploring PerformanceTradeoff in Unstructured P2P Networks, is authored by Kei Ohnishi, Satoshi Nagamatsu and Yuji Oie. In this work, the authors propose a topology reconstruction method that can modify network topologies in a dynamic, autonomous and decentralized way. As a result, the proposed method is able to improve trade-off points between search and access load balancing performance in unstructured Peer-to-Peer (P2P) file sharing networks.

We would like to thank all the authors for the high quality of their papers, the referees for their careful reviews with useful comments and suggestions that improved the quality of this special issue. Thanks also to the International Journal of Hybrid Intelligent Systems Editors, Ajith Abraham and Mario Köppen, for the opportunity and very efficient handling of the publication procedure. 